V.

\title{
Cornual Gestation: Rupture: Pregnancy in Opposite Cornu after Operation.
}

\author{
By Albay Doran, F.R.C.S., \\ Surgeon Samaritan Free Hospital.
}

EARTY in the autumn of 1904 the following case occurred in my operative practice. I removed a cornual sac for acute hæmorrhage due to its rupture, and was able to save the opposite cornu. That cornu, which had already been the seat of two normal pregnancies before the operation, has once more carried a child safely to term since I amputated the cornual sac. I will now relate the case, dwelling afterwards on questions of interest in relation to it, such as the simulation of Hegar's sign, the absence of raginal "show," and the high importance of after-histories in cases of this kind:- -

D.P., aged 27 , applied for relief at the out-patient department of the Samaritan Free Hospital on September 7th, 1904, at 1-30 p.m., suffering from acute abdominal pain with marked anæmia. She was very ill-nourished, being the wife of a day-labourer, residing in the poorest quarter of the Latimer Road district. She had been married for over two years, and borne two children; the youngest, seven months' old, had been weaned three weeks before the patient was brought to the hospital. No show had been seen since the second labour, except on one occasion, when the child was four months old, and the patient believed that she was once more pregnant.

On the evening of September 5th an acute attack of hypogastric pain occurred, and lasted all night. Next morning, when the patient rose, she felt pains distinctly crampy in character, and became faint. The symptoms subsided towards the evening, and on the morning of September 7 th she felt better still, but considered that it was prudent to state her case at a hospital. Mr. A. Lionel Smith noted: "In the middle line of the abdomen is a mass of about the size of a uterus in the fourth month. On vaginal examination the mass in the abdomen is felt to be continuous with the cervix, but the thinning of the supra-vaginal portion of the cervix is much more marked than usual." There had been no "show" of blood during or before the three days" attack of pain. Mr. Lionel Smith induced the patient to rest in the waiting-room for a while. At 5-30 p.m. she felt very faint. At 8-30 p.m. Dr. Cuthbert Lockyer saw her. A severe attack of syncope 
had occurred, and the patient was admitted. At 9 p.m. I was sent for, as she had fainted once more. The mass in the middle line could no longer be defined, and there was dulness over the right side of the abdomen as well as over the hypogastrium. For the first time, a little vaginal "show" was detected. The pulse was 120 , small and thready.

I operated at 9-30 p.m., assisted by Dr. Lockyer, Mr. Lionel Smith administering chloroform. On opening the peritoneal cavity, much fluid blood escaped, with masses of recent clot. I at once seized and clamped a body which looked like the uterus and showed a ragged laceration, about four inches long, on its upper surface towards the left. Placenta and membrane protruded from this laceration, and were traced upwards under the omentum to a fœtus three inches long, lying intact in its amnion. The inner side of the lacerated sac was connected with what I found to be an empty left uterine cornu by a band, free above and continuous with the cervix and pelvic structures inferiorly. The right ovarian vessels were ligatured in the infundibulo-pelvic ligament, and then the band was secured. As all hæmorrhage now ceased, I was enabled to examine the relations of the sac, and found that the right tube ran entire from its outer border to which the ovary was also attached. The sac, shaped somewhat like a paper fruit-bag over four inches square, was internal to the right round ligament which ran from its outer border. The band was transfixed and ligatured with No. 4 China-twist silk and then divided; it became very narrow when the ligature was drawn tight. Thus the ruptured sae came away with the right appendages, whilst the left cornu was saved. I noted that the left tube and ovary were normal. 'l'he peritoneal cavity was freely washed out with saline solution, several pints of which were closed up in that cavity when the abdominal wound was sutured.

There was but little rise of temperature after the operation, and the maximum, $1003^{\circ}$, on the evening of the third day, was apparently due to irritation caused by the presence of scybala in the lower bowel, a common complication after emergency operations. Under the influence of strychnine the pulse rapidly improved. On the second day a discharge of blood from the vagina was noted. It was never free, but bccame foetid and mixed with mucus on the fifth day, without rise of temperature, and disappeared a day or two later. Nothing resembling a decidua was detected in the discharge. After History. When tho patient left the Samaritan Hospital, the period reappeared while she was at a convalescent establishment, and was seen twice again, at regular intervals. Then she became 
pregnant. Labour occurred at term on September 13th, 1905. There was difficulty in micturition during the last three months, and labour was, it appears, impeded by great distension of the bladder. When the catheter was used the labour terminated spontaneously without further trouble. There was no post partum hæmorrhage. The patient has seen no "show" since her last confinement, but she is still suckling her child. Ever since the operation she has been subject to attacks of vertigo, without ever fainting. For this important after-history I was obliged to seek the patient herself, as she had changed her address, but after numerous enquiries in the neighbourhood, I found her (May 3rd, 1906) in a back street in Notting Dale, and for the first time learnt that pregnancy had occurred in the cornu which I was able to save. Her health is fairly good considering her circumstances.

The parts removed will be minutely described by my friend and colleague Dr. Cuthbert. Lockyer, who, I understand, is preparing a monograph on cornual pregnancy, which will be in some respects a continuation of our joint paper on "Two Cases of Uterus Septus Unicollis, both associated with Fibro-myoma, and one also with Trmatosalpinx," which appeared in this JournaL, in March, 1905. At present I need dwell on little more than purely clinical and surgical questions, especially as there can be no doubt that the foetal sac was the right cormu of the uterus.

These clinical and surgical questions will now be discussed in detail :-

1. Intra-peritoneal hæmorrhage was evident, and ectopic gestation was diagnosed. We had little doubt that rupture of the sac had occurred, but the patient's condition was so grave that I made all haste to open the abdomen and stop the hamorrhage without attempting to define the precise nature of the abnormal pregnancy.

2. In this instance of cornual pregnancy no "show" of blood was observed from the beginning of gestation until about half an hour before operation. During the acute symptoms in the course of the last three days before the sac was removed not a trace of blood escaped from the genital canal. After the operation there was a distinct discharge of blood which became foetid. Hæmorrhages from the normal cornu are the rule in cases of pregnancy in a closed cornu. Clinical evidence has shown that the discharge of the decidua from the normal cornu bears little, if any, relation to the progress or arrest of the pregnancy. In tubal gestation discharge of decidua from the uterus is very often associated with arrest of the abnormal pregnancy, and is therefore a valuable monitor to the surgeon. The bloody 
discharge after the operation in this case possibly represented the expulsion of the remains of a decidua long broken down. If so, however, it is strange that a show of blood had not occurred much earlier before operation.

3. As is the rule in cornual and the exception in tubal pregnancy, the patient did not suffer from pain until the rupture took place. The escape of blood into the cavity of a healthy peritoneum causes distinct pain, and therefore explains the earlier acute symptoms in this case. $\mathbf{A}$ few hours later the pains became cramp-like, that is to say, the cornu contracted occasionally, expelling its contents. It did not succeed in emptying its cavity of the entire placenta. As its walls were fairly thick, and made up of uterine muscular tissue, it is easy to understand why these crampy pains set in, and were clearly distinguished and described by the patient.

4. The gravid cornu had risen entirely above the pelvic brim, and thus was exposed to damage. Rupture is rare during the first three months of cornual pregnancy, but more frequent later when the cornu is no longer a little sac lying well protected down in the pelvic cavity.

5. The source of the intra-peritoneal hæmorrhage was manifest. Rupture of the sac is the most frequent cause of interruption of a cornual pregnancy; in tubal pregnancy it is now known to be otherwise. Death of the foetus and its retention in the cornu for an indefinite period has been frequently recorded.

6. One of the most instructive features in this case was the apparent thinning of the supra-vaginal portion of the cervix, which Mr. Lionel Smith carefully noted as being much more marked than in a normal pregnancy. The thin portion was no doubt the band connecting the gravid cornu with its fellow and with the cervix; morphologically the lower part of the band was really cervix. As the gravid cornu had come to lie almost vertically in the middle line, its longitudinal axis was practically continuous with that of the vaginal portion of the cervix. Thus we see how early normal pregnancy may be simulated by cornual gestation, the band giving a false impression of "Hegar's sign" when the cervix is examined by the bimanual method.

7. The foetus measured a little over three inches in length. Making allowance for relative ill-development, the pregnancy might have reached the middle of the third month. Conception during lactation, however, made such calculations very uncertain in this case. The foetus was quite fresh, and must have been alive when the acute symptoms began. Death of the fotus in a cornual sac 
as a rule means its indefinite retention. The familiar results of tubal mole and tubal abortion are not seen in cornual pregnancy.

8. The fact that there was a distinct band between the gravid cornu and the remainder of the uterus is of great interest for surgical reasons. Dr. Lockyer found that the band did not include a canal communicating with the cervix or with the cavity of the normal cornu. As this report is mainly clinical, I need not discuss the question of transmigration of the ovum. I must dwell on the fact that the presence of a well-defined band allows of an essentially conservative operation, the healthy cornu being saved, so that it may become the seat of a normal pregnancy. This happens to be the second case in which I have removed an abnormal cornu from a woman who had borne children in the opposite cornu, and in both instances the normal cornu became the scat of pregnancy after the operation.

The first pationt underwent operation when 38 years of age on Tanuary 3rd, 1899.* I removed an undeveloped right cornu which had become the seat of a myoma. The patient had borne a live child and had also aborted once before the operation. As in the case of cornual pregnancy, I lost sight of the patient and had great difficulty in tracing her, until March, 1902, when she wrote to me, saying: "I had another child in 1901, and a fine big boy be is, now one year and a half old. I had a good quick time; he was born ten minutos before the doctor arrived."

We must bear in mind how very well the normal cornu in a double or one-horned uterus may carry on its functions. "As a rule, the course of pregnancy is not disturbed, and labour is easy and natural," says Professor Stephenson.t So it was in my two cases where the imperfect cornu had been amputated. On that account I consider that the operator is fortunate when he is able to save the unaffected cornu. In subjects where the bifurcation is not complete this conservative procedure may be impossible.

* "The Removal of a Fibroid from a Uterus Unicornis in a Parous Subject." Brit. Med. Joum., Vol. I., 1899, p. 1389. This paper appeared before the patient became pregnant again.

+ Art. : "Pregnancy," Encyclopcedia Medica. 